\title{
A Novel Approach for Mixed Noise Removal using 'ROR' Statistics Combined WITH ACWMF and DPVM
}

\author{
Remya Soman \\ M G University \\ Fisat ,Angamaly \\ Ernakulam Dt. Kerala
}

\author{
Jency Thomas \\ M G University \\ Fisat ,Angamaly \\ Ernakulam Dt.Kerala
}

\begin{abstract}
In this paper a mixed noise removal framework using Robust Outlyingness ratio (ROR) statistics combined with adaptive center weighted median and detail preserving variational approach is discussed. The pixels are classified into different clusters based on the ROR statistics, which measures how impulse like each pixel is .To make the results more accurate, each cluster undergoes coarse and fine stage of noise detection and removal, which make use of ACWMF for noise detection and DPVM for restoration of noise candidates. Final stage of filtering is done by means of Non Local Means filter. Extensive simulations show that the proposed scheme consistently works well in suppressing both impulse and Gaussian noise with different noise ratios.
\end{abstract}

\section{Keywords}

Image Denoising, impulse noise, mixed noise, NLM Filter, ROR statistics.

\section{INTRODUCTION}

Image denoising is one of the promising areas in the digital image processing [1]. Its main objective is to improve the quality of image and get the best estimation from the noisy data. The impulse noise has to be removed to guarantee good performance of image processing tasks like object tracking, edge detection and image segmentation. As it has widely spread applications in the electronic industry, in the field of photography and medical field especially in forensic science where high quality photographs is required to be accepted as evidence, image denoising is thoroughly studied.

Mathematically, if we use $\mathrm{Y}=\mathrm{X}+\mathrm{W}$ to denote the degradation process (X: clean image, $\mathrm{Y}$ : noisy image, $\mathrm{W}$ : noise, the image denoising algorithm attempts to obtain the best estimate of $\mathrm{X}$ from Y.

Images can be represented as binary, gray scale or color images. The binary images are created from the gray scale images take two discrete values black and white. They are also known as pixel images. Gray scale images are one color or monochrome images, which represent brightness of the image. The image has 8 bits per pixel which means it can have 0 to 255 brightness levels; where ' 0 ' represent black and ' 255 'represent white, values from 1 to 254 represents different gray levels. Color images are three band monochrome images. Typical color images are red, green and blue images and are also referred to as RGB images. There are basically two types of noise, additive Gaussian noise and impulse noise. Additive Gaussian noise is statistical noise that has its probability density function equal to that of the normal distribution and is introduced by adding to each image pixel a value with zero mean Gaussian distribution. This means that each pixel in the noisy image is the sum of the true pixel value and a random Gaussian distributed noise value. In the case of Gaussian noise each and every pixel gets affected. Various filtering approaches to remove the noise are linear filtering and non linear filtering. Linear filtering uses mean filter and Least Mean Square (LMS) adaptive filter and non linear filtering is based on Median filter. Mean filter reduces the intensity variation between adjacent pixels and the main application of it comes, when a small area in the image is considered. Linear filtering technique can remove additive Gaussian noise by averaging pixel values. This method is of mathematically simple and offers satisfactory performance on image with additive Gaussian noise. However this type of filtering will blur edges and it is not suitable for impulse noise.

A portion of image pixel is replaced by noise values in the case of impulse noise [2]. Impulse noise can be added to still images in digital cameras or to video sequences in digital television, during image acquisition, recording and transmission .To increase the quality of images in digital cameras and television impulse noise has to be removed. The intensity of impulse noise can be high or low .Salt and Pepper noise also called as fixed valued impulse noise is a kind of impulse noise where each pixel in an image has the probability of $\mathrm{p} / 2(0<\mathrm{p}<1)$ being contaminated by either a white dot (salt) or a black dot (pepper). These noises take either the maximum or minimum pixel value.

\section{$\mathrm{Y}=\{0$ or 255 with probability $\mathrm{p}\}$ \\ $\mathrm{X} \quad$ with the probability $1-\mathrm{p}\}$}

Where $\mathrm{Y}$ represent noisy pixel, $\mathrm{X}$ is the original value.

Random valued impulse noise is uniformly distributed in the interval $[0,255]$. Salt and pepper noise is easier to remove because the difference in gray levels between a noisy pixel and its noise-free neighbors are significant. For impulse noise reduction preserving edges and details is an important problem. Only corrupted pixels should be filtered in order to avoid image smoothing. Impulse noise reduction at the same time detail preservation is an essential requirement for further image processing stages, however achieving these two are difficult. The selection of denoising algorithm is application dependent, which means for example the method of denoising the satellite images may not be used in denoising the medical images. And it is necessary to know the type of noise present in the image in order to select appropriate algorithm for denoising. When the image is corrupted with impulse noise, filtering based approach is proven to be the best. Wavelet based approach of denoising is suitable when the image is corrupted with Gaussian noise.

Non linear filters such as median- based filters [3], are widely exploited due to their improved filtering performance. Using this classical approach impulsive noise could be completely removed. There are other nonlinear filters proposed for impulse noise reduction, like rank-order filters [4], weighted median filters [5], stack filters etc. When compared with linear filters, median filters have an advantage that a single corrupt or noisy pixel in the filtering window will not affect 
the median value much. However these filters are uniformly implemented across the entire image, so they tend to remove the desirable properties. So in order to avoid this problem various detection mechanisms has been introduced prior to filtering and some of these detection schemes are based on previous training, weighted median ,local image statistics [6] ,fuzzy techniques [7] etc. Weighted median filters, center weighted median filters [8] and adaptive center weighted median filters are modified median filters .They give extra weight to some pixels of the filtering window and thus these filters achieve betterment to the standard median filter.

The outline of the paper is as follows, section 2 summarizes various denoising techniques developed so far, gives their advantages and disadvantages. Section 3 describes the proposed method and details of implementation are discussed in section 4 .Simulation results and discussion of the proposed method is discussed in section 5 and section 6 concludes the paper.

\section{RELATED WORKS}

In this section various methods are analyzed to remove both impulse noise and Gaussian noise. Switching strategy $[9,10]$ is one of the best method to avoid the damage of good pixels, where an impulse detector is introduced to determine whether a pixel should be modified or not and the filters based on this strategy are more effective and simple than uniformly applied method such as median filters. Defining a robust decision is difficult since switching filters are based on predefined threshold value and these filters does not take, the local features into account as a result the details and edges may not be recovered satisfactorily .

In [11] Haidi Ibrahim et.al. describe a simple yet efficient way to remove impulse noise from images. This method comprises two stages .In the first stage, pixels are roughly classified either as noisy or noise free pixels. In the second stage only noisy pixels are processed and noise-free pixels are copied into the output image. Based on the number of noise-free pixels, the method adaptively changes the size of the median filter and median is calculated only based on the noise-free pixels. This method is actually a hybrid of adaptive median filter and switching median filter. The main advantage of this method is that it does not need the threshold parameter and hence no training is required.

Median of absolute deviation from the median is modified [12] and used efficiently to separate noisy pixels from the image details. The main advantage of this method is that it does not require previous training and hence a low complex method, also guarantee no sensitivity to image content at low noise ratio. But it tends to remove the details.

Decision based median filters [13-15], take into account both local properties and structure in the image. The pixels are classified either as corrupted or uncorrupted, and then go through the filtering operations. The main problem with the decision based methods lie in building a decision rule or a noise measure that discriminate between corrupted pixels and un corrupted ones.

Yiqiu Dong suggested Directional Weighted median filter [16]. The method includes an impulse detector which is based on the difference between current pixels and neighbors in four main directions. This is combined with weighted median filter for the removal of noise. This method is applied recursively and iteratively with decreasing threshold. It could preserve more details and thin lines, able to restore color images efficiently. But the disadvantage is that it iterates the detection and filtering algorithm minimum 8 to 10 times to give satisfactory results.

Gouchol Pok suggested selective removal of impulse noise based on homogeneity level information [17] for identification of the noise. The idea of homogeneity level is defined and this is based on global and local statistical properties of the pixel. Co-occurrence matrix is used to represent correlation between pixel and its neighbors and to derive upper and lower bound of homogeneity level.

R.Garnett [18] introduced a new statistics Rank Ordered Absolute Differences (ROAD) to detect impulse noise .This ROAD statistics for impulsive noise detection was incorporated into bilateral filter and the result was a trilateral filter which was capable of removing both Gaussian and impulse noise. But the disadvantage found with this method was the ROAD value could be false provided that half of the pixels in the processing window are corrupted. In the case of random valued impulse noise some noise values may be close to their neighbor's value, in which case ROAD value of the pixel may not be large enough for it to be distinguished from the noise free pixels.

To overcome the disadvantages of ROAD a new local image statistics, and hence a new detector was introduced by Y.Dong, R.H .Chan and Shufang Xu [19] which was called Rank Ordered Logarithmic Difference (ROLD) for short. The idea behind that was to increase the difference between noisy pixel and noise free pixels in ROAD so that the detection becomes more accurate. However the method was computationally complex as logarithmic operation is involved.

Raymond H. Chan, proposed a method for removing random valued impulse noise[20], which uses adaptive center weighted median filter to identify pixels which are likely to be corrupted by noise and it uses detail preserving regularization method in [21] and [22] as the restoration method which preserve edges and noise free pixels in a better way. The performance of the combined method is preferred rather than just using ACWMF or DPVM alone, especially when the noise ratio is high.

Effective removal of salt and pepper noise can be done by means of trimmed filter $[23,24]$. The pixel values 0 's and 255 's which are responsible for salt and pepper noise are removed from the image, i.e. the window elements are arranged in the increasing or decreasing order, which makes the removal of the minimum and maximum values easier. Then the median value of the pixels is taken which is used to replace the noisy pixel. However these methods cannot give better result at high noise density. Since most of the pixels will be taking either 0 or 255 at high noise density.

Fuzzy impulse detection [25] is proven to be very efficient among the state-of-the-art denoising algorithms. The main advantage is that it requires no previous training, and more suitable for salt and pepper noise removal, in which case each image pixel has equal probability of being corrupted either to positive impulse ( 255) or a negative impulse (value zero). This impulse detection technique generate a fuzzy flag map , which gives each pixel a fuzzy flag indicating how much it looks like an impulse noise.

Cluster-based Adaptive Fuzzy Switching Median (CAFSM) is a novel technique [26], which composed of cascaded easy to implement, impulse detection mechanism and a detail preserving noise filter. The impulse detector classifies the pixels that are found to be noisy and the filtering phase 
replaces those detected pixels. Filtering phase also employs fuzzy reasoning to deal with uncertainties. CAFSM filter is capable of removing both salt and pepper and random valued impulse noise. The impulse detection can be carried out by analyzing the local image statistics within a window patch whose size is bounded by the filter. The noisy pixels do have intensities vary greatly compared to their neighbors, whereas in the case of noise-free pixels, half of their neighbors have similar intensity. Based on this noisy pixels are classified into one cluster, noise-free pixels in the other and only those pixels which are not noisy are restored. When the images are highly corrupted by noise, discovering the rule base structure becomes quite difficult and that is the disadvantage associated with this.

The approach of Non Local Means (NLM) filtering [27-28] exploits the redundancy present in the image due to the presence of similar patterns. The restoration is done by replacing the pixel intensity with the weighted average of all the pixels in the image. The weight of each pixel depends on the distance between its intensity gray level vector and that of target pixel. The main advantage of NLM filter is that it can remove Gaussian noise effectively but in the case of impulse noise it is difficult since redundancy is less as it is not uniformly distributed. ROR-NLM filter proposed by Bo Xiong [29] separates the noisy pixels into various levels and they are replaced by the median filter. To optimize the performance of non local means filter, some smoothing parameters have to be set manually. In order to set the smoothing parameter automatically a method has been proposed in [30], where they use the Cp statistics in Newton's method to optimize ' $h$ '. This statistic also has the advantage of being a reliable measure of the quality of the denoising process for each pixel.

To achieve robust block classification [31] in noisy images a method based on singular value decomposition and k-means clustering is employed. In this method a local window is adjusted to match the local property of each block and then a rotated matching algorithm is worked for similarity matching. Then using the block classification result and Laplacian operator the noise level is estimated. ANL-means algorithm can effectively identify block with shifted orientation as a close match.

\section{PROPOSED METHOD}

Literature survey reports that there are still open areas for further research and a method is proposed for removing universal noise from digital images and this denoising framework involves ROR, ACWMF detection, DPVM restoration and NL-means filter.

Robust Outlying ness Ratio (ROR) is a new statistics which is used to judge the outlying ness of an observation. ROR measures how much each pixel gets affected by impulse noise. Based on this statistics the pixels are divided into four different clusters and different decision rules are adopted in each clusters for noise detection. During the detection process for better accuracy the from course- to- fine strategy is followed. Different thresholds are used in the two stages and the detection procedure is iteratively adopted. The universal noise filtering framework is based on the Non Local Means .The NL-means is powerful for removing the Gaussian noise, but in the case of impulse noise, the pixels are very different to their neighbors, so exploiting similarity is much difficult. In order to extend the NL-means to remove impulse noise, a reference image and detection result is obtained prior to filtering.
The ROR-NLM filter can be easily extended to remove mixture of Gaussian and impulse noise. An effective solution to remove the mixed noise is to apply the NL-means twice with different values of smoothing parameter ' $h$ ', one with a smaller value to remove the impulse noise and another with a larger value to remove the Gaussian noise , and from the experimental analysis the suitable values are in the interval $[5,10]$.

\subsection{Outline of the Proposed Method}

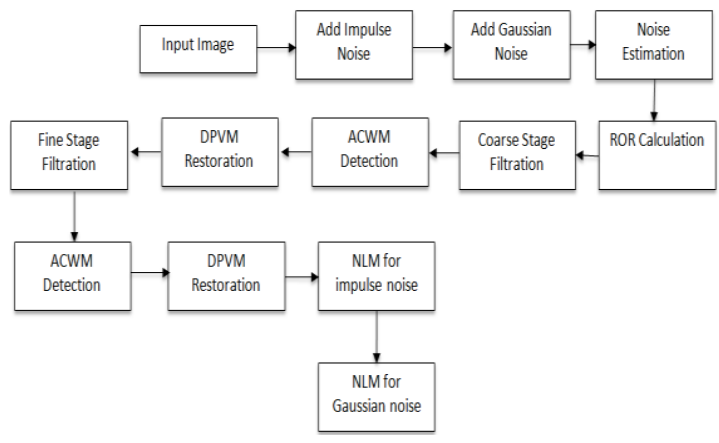

Fig 1: Overall block diagram of the proposed method.

Figure1 represents the overall block diagram of the proposed denoising framework. Based on ROR calculation the pixels are classified into four different clusters/levels. The four levels are the most like level when ROR $>3$, second like level when $2<\mathrm{ROR}<=3$, third like level when $1<\mathrm{ROR}<=2$ and fourth like level $0<\mathrm{ROR}<=1$.Then in each cluster, in order to detect the impulse noise the from coarse-to -fine strategy and the iterative framework is followed. The pixels have different degrees of the impulse like, so different thresholds are adopted in each clusters. Both in coarse and fine stage the detection technique used in ACWM is employed and in the restored procedure of the detection stage detail preserving variation method is used. The thresholds of coarse stage are relatively larger and the thresholds of fine stage are smaller. The output obtained from the fine stage is given to NLM filter for the filtration of both impulse and Gaussian noise.

\section{IMPLEMENTATION DETAILS}

To eliminate the bias created by different manifestations of noise, a standard set of noisy images are created. The standard data set like Lena ,Bridge, Pepper etc form the test image to which different levels of noise is added and these form the common input to each method. In the proposed RORACWM-DPVM-NLM , the pixels of the original, noise free image is classified into four different clusters. In noise detection and filtering the impulse noise and gaussian noise are both considered. The parameters for noise-detection are chosen,based upon the values from experimental analysis.

\subsection{ROR Calculations}

The ROR statistics provide a measure of how impulse-like is a pixel to its neighbors. ROR is not directly used to detect the outliers; it is just used to measure the outlying ness of each pixel in the image.

In order to calculate the ROR, N=2 is used and hence window size is $5 \times 5$. Since the ROR measures the outlying ness of each of the pixels, i.e., a measure of how impulse-like, all pixels are classified into four levels (clusters) according to the value of ROR.The four clusters are the most like cluster for ROR greater than 3; the second like cluster if ROR is between 2 and 3 ; the third like cluster ROR is between 1 and 2 ; and the 
fourth like cluster if ROR is less than 1. The lower the ROR, the lower impulse-like of the pixel in its neighbors.

It is hard to give suitable threshold, since the impulse noise is randomly distributed in an image; the ROR values of the impulse noise are random. When the noise ratio is high, the situation is more complex.

Therefore through experimental analysis the thresholds taken are $\mathrm{T} 1, \mathrm{~T} 2, \mathrm{~T} 3$, and $\mathrm{T} 4[30,40,50,5]$ respectively to represent the thresholds of the most like, second like, third like, and fourth like levels, respectively.

\subsection{Coarse Stage Filteration}

Step 1. Choose the parameters of the algorithm as follows, coarse thresholds and window size $\mathrm{N}($ the actual size is $(2 \mathrm{~N}+1) *(2 \mathrm{~N}+1)$; initial $\mathrm{j}=1$, iterations $\mathrm{mc}$.

Step 2. Initialize the detection flag matrix as zeros, where " 0 s" represent good and "1s" represent noisy pixels.

Step 3. Calculate the ROR of the current pixel. Based on the ROR threshold, noise is detected by means of acwmf method Update the flag matrix according to the result

Step 4.According to the detection result i.e, if the flag is 1, get the Dpvm-based restored image , or do not change.

Step 5. If $\mathrm{j}$ is less than or equal to $\mathrm{mc}$ (no of iterations), $\mathrm{j}=\mathrm{j}+1$ then go to step 2 or the coarse stage is completed.

Through experimental analysis number of coarse iterations is kept as 4 .

\subsection{Fine Stage Filtration}

1. Choose the parameters of the algorithm as follows, fine thresholds and window size $\mathrm{N}($ the actual size is $(2 \mathrm{~N}+1)$ $*(2 \mathrm{~N}+1)$; initial $\mathrm{j}=1$, iterations $\mathrm{mf}$.

2. Initialize the detection flag matrix as zeros, where " 0 s" represent good and "1s" represent noisy pixels.

3. Calculate the ROR of the current pixel. Based on the ROR threshold, noise is detected by means of acwmf method Update the flag matrix according to the result.

4.According to the detection result i.e, if the flag is 1 , get the Dpvm-based restored image , or do not change.

5. If $\mathrm{j}$ is less than or equal to $\mathrm{mf}$ (no of iterations), $\mathrm{j}=\mathrm{j}+1$ then go to step 2 or the fine stage is completed.

Through experimental analysis number of fine iterations when set as 1 , yield better results.

\subsection{Adaptive Center Weighted Median Filter}

In this work, an adaptive center weighted median (ACWM) filter [20] is used in both course and fine stage, for detecting the noise in each cluster s, preserving image details while effectively suppressing impulsive noise. It make use of a novel adaptive operator, that forms the estimates based on the differences between the current pixel and the outputs of center-weighted median(CWM) filters with varied center weights. This employs the switching scheme based on the impulse detection mechanisms. The ultimate output is switched between the median and the current pixel itself.While still using a simple thresholding operation, the proposed filter yields superior results to other switching schemes in suppressing both types of impulses with different noise ratios.

The main steps in ACWM filter are given below.

1. Initialize the noisy image to be observed.

2. Apply ACWM with the 4 thresholds to the noisy image to get the noisy candidates.

3. Using 5 X 5 windows compute Median Absolute Deviation (MAD)

4. The median of absolute deviations from the median (MAD), is a robust estimate of dispersion and its scaled forms are used as the thresholds. Here 0.1 is chosen as the scaling factor $(0 \leq \mathrm{s} \leq 0.6)$.

This choice yields satisfactory results in filtering randomvalued impulse noise when the noise ratio is not high. However, for a high-level noise ratio, the filter cannot preserve the fine features in the images. When the noise ratio is high, ACWMF may falsely detect some noise-free pixels as noisy pixels. And if these erroneous noise candidates form patches, and are located near to edges, it will distort them. To alleviate the problem, one way is to apply the method iteratively with different thresholds.

Assume the window size as $(2 h+1)^{2}$ and $L=2 h(h+1)$.The gray level of the noisy image is denoted by $\mathrm{X}_{\mathrm{ij}}$ at pixel location $(\mathrm{i}, \mathrm{j})$

$$
Y_{i j}^{2 k}=\operatorname{median}\left\{X_{i-u, j-v},(2 k) \oslash X_{i j} \mid-h \leq u, v \leq h\right\}
$$

Where $2 \mathrm{k}$ is the weight given to the pixel $(\mathrm{i}, \mathrm{j})$ and repetition operation is represented by $\diamond$.

$$
d_{k}=\left|Y_{i j}^{2 k}-X_{i j}\right|
$$

In order to determine whether the current pixel $(i, j)$ is corrupted, a set of thresholds $T_{k}$ are employed. If $d_{k}>T_{k}$, $\mathrm{k}=0,1 \ldots . . \mathrm{L}-1$ is true then $\mathrm{X}_{\mathrm{ij}}$ is regarded as noisy candidate.

In the case if $3 * 3$ windows are used (i.e, $\mathrm{h}=1$ and $\mathrm{L}=4$ ), four thresholds $\mathrm{T}_{\mathrm{k}}, \mathrm{k}=0, \ldots \ldots \ldots 3$, are needed. The MAD (median of the absolute deviations) from the median ,which is defined as

$\operatorname{MAD}=\operatorname{median}\left\{X_{i-u, j-v}-Y_{i j}^{0} \mid:-h \leq u, v \leq h\right\}$

This is a robust estimate of dispersion, its scaled forms are used as thresholds.

\subsection{Detail preserving Variational method}

The Dpvm method proposed here uses a non smooth datafitting term ,along with edge preserving regularization. This paper proposes a method to combine ACWM and DPVM for restoring images that are corrupted with random valued impulse noise. In both course and fine stage the noisy pixels are detected using ACWMF, and selective restoration is done on those pixels using DPVM. These two steps are applied alternatively. Furthermore, using regularization method ,the edges and details are preserved for those noise candidates and 
signal candidates do remain the same. The combined approach perform better than just using either ACWM or DPVM .It works efficiently when the noise ratio is as high as $50 \%$.

In DPVM ,the noisy pixels are restored by minimizing a convex objective functional Fy:

$$
\begin{aligned}
& F_{y}(x)=\sum_{(i, j) \in N_{T}}\left|X_{i j}-Y_{i j}\right| \\
& +\frac{\beta}{2}\left(\sum_{(i, j) \in N_{T}} \sum_{(m, n) \in V_{i j}} \varphi \alpha\left(x_{i j}-x_{m n}\right)\right. \\
& +\sum_{+} \sum_{(m, n) \in V_{N_{T}}} \varphi \alpha\left(y_{m n}-x_{i j}\right)
\end{aligned}
$$

\subsection{Non Local Means Filter}

\subsubsection{NLM for impulse noise}

Let us consider the observation model for gaussian noise, $\mathrm{y}=$ $\mathrm{x}+\mathrm{n}$, where, $\mathrm{x} \varepsilon \mathrm{R}^{\mathrm{n}}$ shows the vector representation of the original image, $\mathrm{n}$ represent the gaussian noise with $\sigma \mathrm{I}$ variance and zero mean ,y represent the observed noisy image. The denoised pixel can be derived as weighted average of all the gray values in the image in the set I.

$$
\hat{x}(i)=\sum w(i, j) y(j) / \sum w(i, j)
$$

here weights $w(i, j)$ represent the similarity between neighborhoods of each pair of pixels. But this method cannot be directly used to remove impulse noise ,because impulsive noise pixels are very different to their neighbors, and that do not contain any useful information. So here to deal with this problem, a reference image along with the detection result are taken ,prior using NL means. To apply NL means algorithm some parameters have to be pre given ,smoothing level $h$, patch size $k$, search window W. Through extensive experiments $h=5, k=3, \mathrm{~W}=10$ can be chosen.

\subsubsection{NLM for gaussian noise}

Extending NLM for removing mixture of gaussian and impulse noise can be done easily.Better solution is to locally vary parameters. This require a deep statistical study of ROR statistics, in order to select the parameters automatically. However a simple and effective solution is to apply the NLM twice with two different values of $h$, one with smaller value to remove the impulse noise and another with larger value to remove the gaussian noise. The suitable values are found to be in the interval $[5,10]$.

\section{RESULTS AND DISCUSSIONS}

Simulations are carried out to verify the noise removing capability of ROR-ACWM\&DPVM-NLM filter. Various images at different noise levels are considered, in order to validate the proposed method. The input to the system is the original noise free image. $256 * 256$, 8bit standard gray scale images are considered. Then performance of the filter is evaluated by adding impulse noise ,both salt and pepper and random valued, along with gaussian noise.Then method is evaluated in terms of PSNR value .

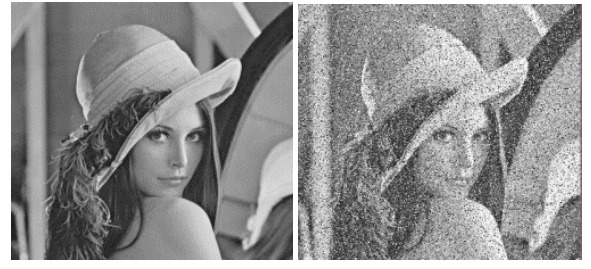

(a)

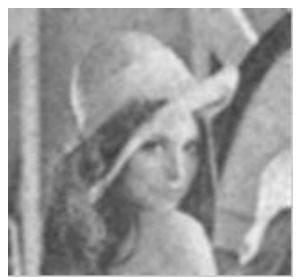

(c) (b)

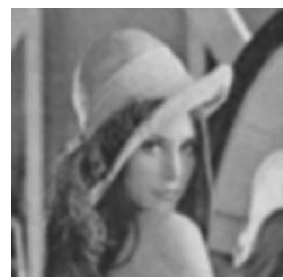

(d)
Fig. 2: An original Lena image (a) noise added image (b) denoised version with ror-nlm(c) and denoised version with the proposed method(d)

The figure $2(a)$ is the standard Lena image, to which $10 \%$ of both impulse and gaussian noise is added, which is represented by 2 (b). The PSNR value of the denoised image 2(c) with ror-nlm is 34.98 , where as with the proposed method ror-acwmf\&Dpvm-nlm 2(d) is 36.7209 .

Table 1. Denoising performance when noise density is $10 \%$

\begin{tabular}{|l|c|c|}
\hline \multicolumn{1}{|c|}{ Image } & $\begin{array}{c}\text { PSNR for NLM } \\
\text { impulse }\end{array}$ & $\begin{array}{c}\text { PSNR for NLM } \\
\text { gaussian }\end{array}$ \\
\hline Lena & 25.4628 & 36.7209 \\
\hline Pepper & 20.4201 & 32.961 \\
\hline Cameraman & 20.1497 & 32.6289 \\
\hline
\end{tabular}

Table 1 shows the result of proposed method when noise density of $10 \%$ is added. Standard data set Lena ,Pepper and Cameraman is considered for evaluation. The result shows that the filter works satisfactorily for mixed noise. The PSNR value obtained after removing impulse noise alone from the image Lena is 25.4628 , then the filter is reapplied to remove gaussian noise as well ,the PSNR value obtained after removing gaussian noise is 36.7209 .

Table 2. Denoising performance when noise density is $\mathbf{5 0 \%}$

\begin{tabular}{|l|c|c|}
\hline \multicolumn{1}{|c|}{ Image } & $\begin{array}{c}\text { PSNR for NLM } \\
\text { impulse }\end{array}$ & $\begin{array}{c}\text { PSNR for NLM } \\
\text { Gaussian }\end{array}$ \\
\hline Lena & 26.0133 & 39.2762 \\
\hline Pepper & 19.9971 & 33.738 \\
\hline Cameraman & 19.8013 & 33.4001 \\
\hline
\end{tabular}

Table 2 shows the denoising result when noise density is $50 \%$. The performance of the filter is much better when applied to high noise density.

\section{CONCLUSION}

Image denoising is an important area of research as it has potential applications in a number of different areas such as 
electromagnetic imaging of objects, medical diagnostics, remote sensing, robotics, etc. The problem taken for this work is to develope a frame work that suppress both impulse and gaussian noise, with high efficiency, low distortion , less computational complexity and less run time overhead. In this paper a method is proposed by combining Robust Outlying ness ratio (ROR) which measures how impulse like each pixel is, with adaptive center weighted median filter (ACWMF) and Detail Preserving Variation Method (DPVM). This universal noise removing framework is capable of removing both impulse and Gaussian noise .Most of the denoising algorithms are either for removing impulse noise or gaussian noise and this work concentrate mainly on the removal of mixed noise. The overhead of using clustering algorithm is not incurred as clusters are formed based on the simple statistics ROR. In order to increase the accuracy, the iterative framework of the from coarse to fine strategy is followed ,the usage of DPVM as the restoration method in both the coarse and fine stage increases the efficiency as it can restore large patches of noisy pixels because of the pertinent prior information via the regularization term. The experimental results prove that the method work exceedingly well when the noise ratio is high as $50 \%$.

Assuming the detection work as pixel wise, the proposed method is implemented to the pixels one by one. As a trial work a new detection pattern can be proposed that detects all the noisy pixels once in the block, and this method could be called as the block-wise pattern.

\section{REFERENCES}

[1] Rafael C. Gonzalez, Richard E. Woods, "Digital Image Processing" Prentice-Hall, India, second edition, 2007.

[2] I. Pitas and A. N. Venetsanopoulos, Nonlinear Digital Filters: Principles and Applications. Norwell, MA:Kluwer, 1990.

[3] Y. H. Lee and S. A. Kassam, "Generalized median filtering and related nonlinear filtering techniques," IEEE Trans. Acoust., Speech, Signal Processing, vol. ASSP33, pp. 672-683, June 1985.

[4] Igor Aizenberg "Effective Impulse Detector Based on Rank-Order Criteria " IEEE Signal Process. Letter., vol. 11, no. 3, Mar. 2004.

[5] D. Brownrigg, "The weighted median filter," Commun. $A C M$, vol. 27, no. 8, pp. 807-818, Aug. 1984.

[6] D. Florencio and R.W. Schafer, "Decision-based median filter using local signal statistics," Proc. SPIE, vol. 2308, pp. 268-275, Sept. 1994.

[7] F. Russo and G. Ramponi, "A fuzzy filter for images corrupted by impulse noise," IEEE Signal Process. Lett., vol. 3, no. 6, pp. 168-170, 1996.

[8] S. J. Ko and Y. H. Lee, "Center weighted media filters and their applications to image enhancement "IEEE Trans. Circuits Syst., vol. 38, no. 9, pp. 984-993, Sep. 1991.

[9] T. Chen and K. Ma and L. Chen, "Tri-state median filter for image denoising," IEEE Trans. Image Process., vol. 8, no. 12, pp. 1834-1838, 1999.

[10] T. Sun and Y. Neuvo, "Detail-preserving median based filters in image processing," Pattern Recognit. Lett., vol. 15, pp. 341-347, Apr. 1994.
[11] Haidi Ibrahim, Nicholas Sia Pik Kong "Simple Adaptive Median Filter for the Removal of Impulse Noise from Highly Corrupted Images", IEEE Transactions on Consumer Electronics, Vol. 54, No. 4, NOVEMBER 2008.

[12] V. Crnojevic, V. Senk, and Z. Trpovski, "Advanced impulse detection based on pixel-wise MAD," IEEE Signal Process. Lett., vol. 11, no. 7,pp. 589-592, Jul. 2004.

[13] C.-T. Chen and L.-G. Chen, "A self-adjusting weighted median filter for removing impulse noise in image," in Proc. IEEE Int. Conf. on Image Processing, 1998, pp. 419-422.

[14] D. A. F. Florencio and R. W. Schafer, "Decision-based median filter using local signal statistics," Proc. SPIE, vol. 2308, pp. 268-275, 1994.

[15] T. Kasparis, N. S. Tzannes, and Q. Chen, "Detailpreserving adaptive conditional median filters," $J$. Electron. Imag., vol. 1, no. 14, pp. 358-364, 1992.

[16] Y. Dong an S. Xu "A new directional weighted median filter for removal of random-value impulse noise " IEEE Signal Process. Letter., vol. 14, no. 3, pp. 193-196, Mar. 2007.

[17] Pok, J. C. Liu, and A. S. Nair, "Selective removal of impulse noise based on homogeneity level information," IEEE Trans. Image Process., vol. 12, no. 1, pp. 85-92, Jan. 2003.

[18] R. Garnett, T. Huegerich, C. Chui, and W. J. He, "A universal noise removal algorithm with an impulse detector," IEEE Trans. Image Process., vol. 14, no. 11, pp. 1747-1754, Nov. 2005.

[19] Y. Dong, R. H. Chan, and S. Xu, "A detection statistic for random valued impulse noise," IEEE Trans. Image Process., vol. 16, no. 4, pp. 1112-1120, Mar. 2007.

[20] Raymond H.Chan , Chen $\mathrm{Hu}$ and Mila Nikolova, "An iterative Procedure for removing random-valued impulse noise. ," IEEE Signal Process. Letter., vol. 11, no.12, pp. 921-924, Dec. 2004.

[21] M. Nikolova, "A variational approach to remove outliers and impulsenoise," J. Math. Imag. Vis., vol. 20, pp. 99 120, 2004.

[22] M. Nikolova, "Minimizers of cost-functions involving nonsmooth data-fidelity terms. Application to the processing of outliers," SIAM J. Numer. Anal., vol. 40, pp. 965-994, 2002.

[23] K. Aiswarya, V. Jayaraj, and D. Ebenezer, "A new and efficient algorithm for the removal of high density salt and pepper noise in images and videos," in Second Int. Conf. Computer Modeling and Simulation,2010, pp. 409-413.

[24] S. Esakkirajan, T. Veerakumar, Adabala N. Subramanyam, and C. H. PremChand "Removal of High Density Salt and Pepper Noise Through Modified Decision Based unsymmetricTrimmed Median Filter "IEEE Signal Process. Letter., vol. 18, no.5, May. 2011.

[25] W. Luo, "A new efficient impulse detection algorithm for the removal of impulse noise," IEICE Trans. Fundam. Electron., Commun., Comput., vol. E88-A, no. 10 , pp. 
[26] Kenny Kal Vin Toh and Nor Ashidi Mat Isa "ClusterBased Adaptive Fuzzy Switching Median Filterfor Universal Impulse Noise Reduction" IEEE Transactions on Consumer Electronics, Vol. 56, No. 4, November 2010 .

[27] Subhojit Sarker,Shalini Chowdhary ,Samanwita laha and Debika Dey "Use of Non Local Means Filter to Denoise Image Corrupted by salt and pepper noise " Signal \& Image Processing : An International Journal (SIPIJ), Vol .3 ,No.2,April 2012

[28] J. Dinesh Peter, V. K. Govindan, and A. T. Mathew, "Robust estimation approach for nonlocal-means denoising based on structurally similar patches," Int. J. Open Problems Compt. Math., vol. 2, no. 2, June 2009.

[29] Bo Xiong and Zhouping Yin, "A Universal Denoising Framework with a new impulse Detector and Non local
Means :IEEETrans. Image processing ,vol21, no4 ,April 2012,pp1663-1675.

[30] Vincent Doré and Mohamed Cheriet, "Robust NL-Means Filter With Optimal Pixel-Wise Smoothing Parameter for Statistical Image Denoising," IEEE Trans.signal Process., vol. 57, no. 5, pp. 1703-1716, May. 2009.

[31] Tanaphol Thaipanich, Byung Tae Oh, Ping-Hao Wu, Daru Xu and C.-C. Jay Kuo, "Improved Image Denoising with Adaptive Nonlocal Means(ANL-Means) Algorithm", The International Conference on Consumer Electronics 2010 (ICCE2010)for image denoising," IEEE Trans. Image Process., vol. 8, no. 12, pp. 1834-1838, 1999. 